\title{
Efficient Graph Packing via Game Colouring
}

\author{
H. A. KIERSTEAD ${ }^{1 \dagger}$ and A. V. KOSTOCHKA $2 \ddagger$ \\ ${ }^{1}$ Department of Mathematics and Statistics, Arizona State University, Tempe, AZ 85287, USA \\ (e-mail: kierstead@asu.edu) \\ ${ }^{2}$ Department of Mathematics, University of Illinois, Urbana, IL 61801, USA \\ and \\ Institute of Mathematics, Novosibirsk, Russia \\ (e-mail: kostochk@math.uiuc.edu)
}

Received 4 August 2008; revised 27 October 2008; first published online 22 May 2009

\begin{abstract}
The game colouring number $\operatorname{gcol}(G)$ of a graph $G$ is the least $k$ such that, if two players take turns choosing the vertices of a graph, then either of them can ensure that every vertex has fewer than $k$ neighbours chosen before it, regardless of what choices the other player makes. Clearly $\operatorname{gcol}(G) \leqslant \Delta(G)+1$. Sauer and Spencer [20] proved that if two graphs $G_{1}$ and $G_{2}$ on $n$ vertices satisfy $2 \Delta\left(G_{1}\right) \Delta\left(G_{2}\right)<n$ then they pack, i.e., there is an embedding of $G_{1}$ into the complement of $G_{2}$. We improve this by showing that if $\left(\operatorname{gcol}\left(G_{1}\right)-1\right) \Delta\left(G_{2}\right)+\left(\operatorname{gcol}\left(G_{2}\right)-1\right) \Delta\left(G_{1}\right)<n$ then $G_{1}$ and $G_{2}$ pack. To our knowledge this is the first application of colouring games to a non-game problem.
\end{abstract}

\section{Introduction}

The purpose of this article is to demonstrate that game colouring can be a useful tool for attacking a non-game problem. We will use optimal strategies for Alice in the marking game to obtain an efficient packing of two graphs. It is our expectation that there will be other applications of this natural technique. A secondary goal is to illustrate a paradigm for generalizing results concerning graphs with bounded maximum degree.

The paper is organized as follows. In Sections 2, 3 and 4 we define and briefly discuss generalized colouring numbers, chromatic and marking games, and graph packing. In Section 5 we state and prove our main theorem. In Section 6 we make some concluding remarks.

$\dagger$ Research supported in part by NSA grant H98230-08-1-0069.

$\ddagger$ Research supported in part by NSF grant DMS-0650784 and by grant 06-01-00694 of the Russian Foundation for Basic Research. 
The following graph $Q_{t}$ will be used as an example several times. It is obtained from $K_{t}$ by first duplicating edges so that every original edge has multiplicity $t$ and then subdividing every edge exactly once. The $t$ original vertices of $K_{t}$ are called branch vertices of $Q_{t}$; the remaining $t\left(\begin{array}{l}t \\ 2\end{array}\right)$ vertices are called subdivision vertices.

\section{Generalized colouring numbers}

Fix a graph $G=(V, E)$ and denote the set of linear orderings on $V$ by $\Pi$. Recall that the colouring number $\operatorname{col}(G)$ of $G$ is the least integer $k$ such that, for some $L \in \Pi$, every vertex has fewer than $k$ neighbours that precede it in $L$. It is well known that $\operatorname{col}(G) \leqslant d$ if and only if every subgraph has a vertex with degree less than $d$. This observation provides a polynomial-time algorithm for determining $\operatorname{col}(G)$.

Starting with Chen and Schelp, several authors $[6,13,11,14]$ have considered useful variations of colouring number, with names such as arrangeability, admissibility and rank. Kierstead and Yang [16] standardized these ideas in terms of a hierarchy of colouring numbers as follows. Let $L \in \Pi$. A vertex $z$ is $k$-reachable from a vertex $x$ with respect to $L$ if and only if $z<_{L} x$ and there exists an $x, z$-path $P$ of length at most $k$ such that $x<_{L} y$ for all interior vertices $y$ of $P$. Let $R_{L, k}(x)$ denote the set of vertices that are $k$-reachable from $x$ with respect to $L$. The $k$-colouring number was defined in [16] to be

$$
\operatorname{col}_{k}(G)=1+\min _{L \in \Pi} \max _{x \in V} R_{L, k}(x) .
$$

The 1-colouring number of $\mathrm{G}$ is just the ordinary colouring number. The following easy inequalities hold for $j \leqslant k$ :

$$
\chi(G) \leqslant \operatorname{col}(G) \leqslant \operatorname{col}_{j}(G) \leqslant \operatorname{col}_{k}(G) \leqslant \Delta(G)(\Delta(G)-1)^{k-1}+1 .
$$

The graph $Q_{t}$ satisfies $\operatorname{col}\left(Q_{t}\right)=3$. Each of arrangeability, admissibility and rank can be polynomially bounded in terms of 2-colouring number and vice versa, but for specific applications each may have its own advantage. However, the 2-colouring number cannot be bounded in terms of the colouring number, since $\operatorname{col}_{2}\left(Q_{t}\right)=t+1$. Ordering all branch vertices before all subdivision vertices witnesses the upper bounds on both $\operatorname{col}_{2}\left(Q_{t}\right)$ and $\operatorname{col}\left(Q_{t}\right)$. For the lower bound on $\operatorname{col}_{2}\left(Q_{t}\right)$, note that for every ordering $L$, if $x$ is the last branch vertex then $\left|R_{L, 2}(x)\right| \geqslant t$.

The 2-colouring number will have special importance for us. It - or slight variations of it - has been previously used in several different contexts. In a typical application one would like to show that a conclusion about the class of graphs with bounded maximum degree holds for the larger class of graphs with bounded colouring number. When we are unable to do this we may still be able to derive the conclusion for the intermediate class of graphs with bounded 2-colouring number. Here is the original example. Burr and Erdős [4] made the following conjecture.

Conjecture 2.1 ([4]). For every positive integer $k$ there exists an integer $C$ such that, for every graph $G$ on $n$ vertices with $\operatorname{col}(G) \leqslant k$ and every graph $H$ on $C n$ vertices, either $G \subseteq H$ or $G \subseteq \bar{H}$. 
Chvátal, Rödl, Szemerédi and Trotter [7] proved a weaker version of the conjecture with $\Delta(G) \leqslant k$ instead of $\operatorname{col}(G) \leqslant k$. Then Chen and Schelp [6] introduced the original precursor of the 2-colouring number, and showed (essentially) that the conjecture holds with $\operatorname{col}_{2}(G) \leqslant k$. They demonstrated the significance of their result by showing (essentially) that the 2-colouring number of planar graphs is bounded, even though their maximum degree is not. Kierstead and Trotter [13] showed that it is bounded by 10, and gave an example to show that it is at least 8. A simpler example is the 'buckyball', i.e., the dual of the graph formed by a 32-panel football, or soccer ball (drawn with the stitching). To see this, fix an order $L$ on the vertices and let $x$ be the largest vertex that has a larger neighbour. After noting that if $y$ and $x$ have a common neighbour larger than $x$ then $y \in R_{L, 2}(x)$, it easily follows that $\left|R_{L, 2}(x)\right| \geqslant 7$. Very recently Kierstead, Mohar, Śpacapan, Yang and $\mathrm{Zhu}[12]$ proved the following theorem.

Theorem 2.2 ([12]). Every planar graph $G$ satisfies $\operatorname{col}_{2}(G) \leqslant 9$.

A vertex colouring of $G$ is degenerate if the union $U$ of any $k$ colour classes satisfies $\operatorname{col}(G[U]) \leqslant k$. In particular, setting $k=1$ we see that a degenerate colouring is a proper colouring; moreover, setting $k=2$ we see that it is an acyclic colouring. The degenerate chromatic number $\chi_{d}(G)$ of $G$ is the least $k$ such that $G$ has a degenerate colouring with $k$ colours. While $\operatorname{col}\left(Q_{t}\right)=3$, we have $\chi_{d}(G) \geqslant \chi_{a}\left(Q_{t}\right)=t$. If we try to use fewer colours then two branch vertices will have the same colour, and two of their common neighbours will also share a colour, creating a bicoloured 4-cycle. However, $\chi_{d}(G) \leqslant \Delta(G)(\Delta(G)-1)+1$. Fix an order $L$, and colour sequentially so that each new vertex $x$ gets a colour that has has not been used on $\left|R_{L, 2}(x)\right|$. Then each vertex $y$ will have at most one neighbour that precedes it in each colour class. This idea leads to the following observation [12], as well as its list colouring counterpart.

Theorem 2.3. Every graph $G$ satisfies $\chi_{d}(G) \leqslant \operatorname{col}_{2}(G)$. In particular, $\chi_{d}(G) \leqslant 9$, if $G$ is planar.

Finally, we remark that generalized colouring numbers are related to the concept of bounded expansion classes (see Nešetřil and Ossona de Mendez [18]). Zhu [24] has very recently proved that a graph class $\mathcal{K}$ has bounded expansion if and only if there exists a function $f$ such that every graph $G \in \mathcal{K}$ satisfies $\operatorname{col}_{k}(G) \leqslant f(k)$ for all natural numbers $k$.

\section{Games}

The chromatic game is played on $G$ by two players, Alice and Bob, with a fixed set of colours $C$. The game begins with Bob deciding which player will make the first move. Once this decision is made, the players take turns colouring the vertices with colours from $C$ with legal colours, i.e., so that adjacent vertices receive distinct colours. Alice wins if the graph is eventually properly coloured; Bob wins if at some time there is an uncoloured vertex that cannot be legally coloured. The game chromatic number $\chi_{g}(G)$ of $G$ is the least integer $k$ such that Alice has a winning strategy when $|C|=k$; for a class $\mathcal{C}$ of graphs 
$\chi_{g}(\mathcal{C})=\max _{G \in \mathcal{C}} \chi_{g}(G)$. This game was originally introduced by Brams and published by Gardner [10] for planar graphs. It was re-invented and introduced to the mathematical community by Bodlaender [1]. Faigle, Kern, Kierstead and Trotter [9] showed that the game chromatic number of the class of forests is 4 . Kierstead and Trotter [13] used a variant of the 2-colouring number to show that the game chromatic number of planar graphs is at most 33. This result was improved by a series of papers $[8,21,11,23]$. The following bound obtained by $\mathrm{Zhu}$ is currently the best.

Theorem 3.1 ([23]). Every planar graph $G$ satisfies $\chi_{g}(G) \leqslant 17$.

Proofs in $[9,21,11,23]$ are all obtained by bounding a new game parameter (implicitly in [9]), the game colouring number. The marking game is played by Alice and Bob on G. Bob decides who goes first and then the players take turns choosing unchosen vertices until all vertices have been chosen. Let $L$ be the order in which the vertices are chosen, where the first vertex is smallest. The score of the game is the maximum $k$ such that in $L$ some vertex has $k-1$ smaller neighbours. Alice's goal is to keep the score low, while Bob's goal is to force it high. The game colouring number $\operatorname{gcol}(G)$ is the least $k$ such that Alice can always obtain a score of at most $k$. The game $k$-colouring number $\operatorname{gcol}_{k}(G)$ of $G$ is defined analogously. Clearly $\operatorname{gcol}(G)=\Delta(G)+1$ if $G$ is regular. Moreover, if Alice interprets Bob's moves in the chromatic game as choices of vertices in the marking game, then she can choose vertices to colour so that no vertex has $\operatorname{gcol}(G)$ coloured neighbours. It follows that:

$$
\chi(G) \leqslant \operatorname{col}(G) \leqslant \operatorname{gcol}(\mathrm{G}) \leqslant \Delta(G)+1 \text { and } \chi_{g}(G) \leqslant \operatorname{gcol}(G) .
$$

However, $\operatorname{gcol}(G)$ cannot be bounded in terms of $\operatorname{col}(G)$, since $\operatorname{col}\left(Q_{t}\right)=3$, but $\operatorname{gcol}\left(Q_{t}\right)>$ $s=\log _{2}(t)$ for $t=2^{s}$. Argue by induction on $s$ and observe that the base step $s=0$ is trivial. First Bob chooses $\frac{1}{2} t$ subdivision vertices that dominate all branch vertices. Alice can respond by choosing at most $\frac{1}{2} t$ branch vertices, and so there exists a set $U$ of $\frac{1}{2} t$ unchosen branch vertices, each of which is adjacent to a chosen subdivision vertex. Now apply the induction hypothesis to $G[U]$. The following theorem is the third example of our paradigm.

Theorem 3.2 ([11]). Every graph $G$ satisfies $\operatorname{gcol}(G) \leqslant 3 \operatorname{col}_{2}(G)-1$.

Kierstead and Trotter [14] showed that if $G$ is planar then $\operatorname{col}_{4}(G)$ is bounded. They used this to show that $\operatorname{gcol}_{2}(G)$ is bounded, which was key in showing that the game oriented chromatic number of $G$ is bounded. Kierstead and Yang [16] showed that the game $k$-colouring number is bounded in terms of the $2 k$-colouring number and that the $k$-colouring number of planar graphs is bounded.

\section{Packing}

Two $n$-vertex graphs $G_{1}$ and $G_{2}$ pack if there exists an edge-disjoint placement of these graphs onto the same set of $n$ vertices. By definition, $G_{1}$ and $G_{2}$ pack if $G_{1}$ is a subgraph of the complement $\bar{G}_{2}$ of $G_{2}$. A number of basic graph-theoretic problems can be expressed in a unified and symmetric form as graph packing problems. Among such problems are 
Turán-type, Ramsey-type, colouring and equitable colouring problems. Since even partial cases of graph packing problems are NP-hard, study of extremal problems was initiated in 1970s by seminal papers of Bollobás and Eldridge [2], Sauer and Spencer [20], and Catlin [5]. Their conditions involved maximum degrees of graphs. For example, Sauer and Spencer [20] proved the following result.

Theorem 4.1 ([20]). If $G_{1}$ and $G_{2}$ are graphs on $n$ vertices and $2 \Delta\left(G_{1}\right) \Delta\left(G_{2}\right)<n$, then $G_{1}$ packs with $G_{2}$.

There have been several refinements of Theorem 4.1. One of them involves the maximum edge degree $\theta(G):=\max _{x y \in E(G)}(d(x)+d(y))$. Clearly, $\theta(G)$ is closely related to the maximum degree of the line graph $L(G)$,

$$
\theta(G)=\Delta(L(G))+2
$$

and $\theta(G) \leqslant 2 \Delta(G)$ for every graph $G$. Kostochka and $\mathrm{Yu}$ [17] showed that $2 \Delta\left(G_{1}\right)$ could be replaced by $\theta\left(G_{1}\right)$ in Theorem 4.1, as well as the following result.

Theorem 4.2 ([17]). If two n-vertex graphs $G_{1}$ and $G_{2}$ satisfy the inequality $\theta\left(G_{1}\right) \Delta\left(G_{2}\right) \leqslant$ $n$, then $G_{1}$ and $G_{2}$ pack, with the following exceptions:

(I) $G_{1}$ is a perfect matching and $G_{2}$ either is $K_{n / 2, n / 2}$ with $n / 2$ odd or contains $K_{n / 2+1}$,

(II) $G_{2}$ is a perfect matching, and $G_{1}$ either is $K_{r, n-r}$ with $r$ odd or contains $K_{n / 2+1}$.

Bollobás, Kostochka and Nakprasit [3] showed that if one of the two graphs has small colouring number then much weaker conditions on $\Delta\left(G_{1}\right)$ and $\Delta\left(G_{2}\right)$ imply the existence of a packing.

Theorem 4.3 ([3]). Let $\operatorname{col}\left(G_{1}\right) \geqslant 3$. If $G_{1}$ and $G_{2}$ are graphs on $n$ vertices with

$$
40 \Delta\left(G_{1}\right) \ln \Delta\left(G_{2}\right)<n \text { and } 40 \operatorname{col}\left(G_{1}\right) \Delta\left(G_{2}\right)<n,
$$

then $G_{1}$ packs with $G_{2}$.

Let $G_{1}$ and $G_{2}$ be graphs on $n$ vertices. We could write the hypothesis of Theorem 4.1 as a two-term sum. Then motivated by Theorem 4.3 and our paradigm, we might consider strengthening Theorem 4.1 to

$$
\exists C C \operatorname{col}\left(G_{1}\right) \Delta\left(G_{2}\right)+C \operatorname{col}\left(G_{2}\right) \Delta\left(G_{1}\right)<n \text { implies } G_{1} \text { packs with } G_{2} .
$$

This is too rash to call a conjecture, and we certainly cannot prove it, but the following weakening according to our paradigm is a corollary to our main result, Theorem 5.1.

Corollary 4.4. Let $G_{1}$ and $G_{2}$ be graphs on $n$ vertices. If

$$
3 \operatorname{col}_{2}\left(G_{1}\right) \Delta\left(G_{2}\right)+3 \operatorname{col}_{2}\left(G_{2}\right) \Delta\left(G_{1}\right)<n
$$

then $G_{1}$ packs with $G_{2}$. 


\section{The main result}

Our main result, Theorem 5.1, proved in this section, not only is a natural example of our paradigm, but also, to our knowledge, is the first use of game colouring to prove a non-game result. The statement of the theorem is stronger than that of the Sauer-Spencer theorem, since $\operatorname{gcol}(G)-1 \leqslant \Delta(G)$ for every graph $G$. The proof is motivated by the classical back-and-forth construction, showing that any two countable dense linear orders without endpoints are isomorphic.

Theorem 5.1. If $G_{1}$ and $G_{2}$ are n-vertex graphs and

$$
\left(\operatorname{gcol}\left(G_{1}\right)-1\right) \Delta\left(G_{2}\right)+\left(\operatorname{gcol}\left(G_{2}\right)-1\right) \Delta\left(G_{1}\right)<n,
$$

then $G_{1}$ and $G_{2}$ pack.

Proof. For $i \in[2]$ set $G_{i}:=\left(V, E_{i}\right), d_{i}(v):=d_{G_{i}}(v), g_{i}:=\operatorname{gcol}\left(G_{i}\right)-1$ and $\Delta_{i}=\Delta\left(G_{i}\right)$. A packing of $G_{1}$ with $G_{2}$ will be viewed as a bijection $f: V \rightarrow V$ such that every $u v \in E_{1}$ satisfies $f(u) f(v) \notin E_{2}$.

Our plan is to construct a packing of $G$ in stages. At the end of stage $s$ we will have a partial packing $f^{s}$ of $H_{1}^{s} \subseteq G_{1}$ onto $H_{2}^{s} \subseteq G_{2}$, where $\left|H_{i}^{s}\right|=s$. We will maintain the invariant $H_{i}^{s} \subseteq H_{i}^{t}$, but not necessarily $f^{s} \subseteq f^{t}$, for $s<t$. At stage $s$ we will choose two new vertices $x_{1}^{s}$ and $x_{2}^{s}$ with $x_{i}^{s} \in V\left(G_{i}-H_{i}\right)$. These choices will be interpreted as moves in two colouring games, Game ${ }_{1}$, played on $G_{1}$, and $\mathrm{Game}_{2}$, played on $G_{2}$. The players for Game $_{i}$ are Alice ${ }_{i}$ and $\mathrm{Bob}_{i}$, although as we shall see it would perhaps be more informative to change the names to Algorithm and Black Box. We assume that each Alice ${ }_{i}$ plays with an optimal strategy witnessing the game colouring number of $G_{i}$. We will provide each $\mathrm{Bob}_{i}$ with a strategy designed to produce a packing.

Recall that $\mathrm{Bob}_{i}$ decides who plays first. For our purposes, we have $\mathrm{Bob}_{i}$ choose to play first if and only if $i=2$. Let $x_{i}^{1}, x_{i}^{2}, \ldots, x_{i}^{n}$ be the sequence of moves in $\mathrm{Game}_{i}$; so $\mathrm{Bob}_{i}$ plays $x_{i}^{s}$ if and only if $i+s$ is odd. The optimal strategy of Alice $i_{i}$ must be able to handle this situation. At stage 1 of the construction Alice ${ }_{1}$ chooses $x_{1}^{1}$ in accordance with her optimal strategy. Our construction will generate $f^{1}\left(x_{1}^{1}\right)=x_{2}^{1}$, which is interpreted as Bob ${ }_{2}$ 's first play in Game 2 . At stage 2, Alice 2 responds to $\mathrm{Bob}_{2}$ by choosing $x_{2}^{2}$ in accordance with her optimal strategy. Then the construction will generate $\left(f^{2}\right)^{-1}\left(x_{2}^{2}\right)=x_{1}^{2}$, which is interpreted as Bob ${ }_{1}$ 's response to $x_{1}^{1}$. Continuing in this back-and-forth fashion, we will generate the two sequences $x_{i}^{1}, x_{i}^{2}, \ldots, x_{i}^{n}, i \in[2]$. By the definition of game colouring number, Alice ${ }_{i}$ 's optimal strategy ensures that the set $N_{i}^{+}(w)$ of neighbours of $w$ preceding $w$ in $H_{i}^{s}$ has cardinality at most $g_{i}$. It remains to show how the construction will generate $x_{i}^{s}$ for $i+s$ odd.

Argue by induction on $s$. The base step $s=0$ is trivial, so consider the induction step $s+1$. By the induction hypothesis, $f^{s}$ is a packing of $H_{1}^{s}$ with $H_{2}^{s}$. Assume $s+1$ is odd as the even case is symmetrical. So Alice ${ }_{1}$ has just chosen $x:=x_{1}^{s+1}$. For $x^{\prime} \in V\left(G_{2}-H_{2}^{s}\right)$ define the set $C\left(x^{\prime}\right)$ by

$$
C\left(x^{\prime}\right):=\left\{y \in H_{1}^{s}: x y \in E_{1} \text { and } x^{\prime} f^{s}(y) \in E_{2}\right\} .
$$


Case 1. $C\left(x^{\prime}\right)=\emptyset$ for some $x^{\prime} \in V\left(G_{2}-H_{2}^{s}\right)$.

Setting $x_{2}^{s+1}:=x^{\prime}$ and $f^{s+1}=f^{s} \cup\left\{\left(x, x^{\prime}\right)\right\}$, we are done.

Case 2. $C\left(x^{\prime}\right) \neq \emptyset$ for all $x^{\prime} \in V\left(G_{2}-H_{2}^{s}\right)$.

Fix $x^{\prime} \in V\left(G_{2}-H_{2}^{s}\right)$; set $x_{2}^{s+1}:=x^{\prime}$ and $C:=C\left(x^{\prime}\right)$. We will show that for some good $z \in V\left(H_{1}^{s+1}\right) \backslash C$, the mapping $\varphi:=\varphi_{z}$ obtained from $f:=f^{s} \cup\left\{\left(x, x^{\prime}\right)\right\}$ by switching the images of $x$ and $z$ is a packing of $H_{1}^{s+1}$ with $H_{2}^{s+1}$. Suppose $z \in V\left(H_{1}^{s+1}\right) \backslash C$ is bad, i.e., not good. Then there exists an edge $u v \in E_{1}$ with $\varphi(u) \varphi(v) \in E_{2}$. Since $f^{s}$ packs $H_{1}^{s}$ with $H_{2}^{s}$, at least one, say $v$, of $u, v$ is in $\{x, z\}$. Since $z \notin C$, we have $u \notin\{x, z\}$.

Suppose $v=x$. Then $x u \in E_{1}$ and $\varphi(x) \varphi(u)=f^{s}(z) f^{s}(u) \in E_{2}$. Thus $f^{s}(z) \in P$, where

$$
P:=\bigcup_{u \in N_{1}^{+}(x)} N_{2}\left(f^{s}(u)\right) .
$$

Since $\left|N_{1}^{+}(x)\right| \leqslant g_{1}$ and $\left|N_{2}(w)\right| \leqslant \Delta_{2}$ for all $w \in V$, we have $|P| \leqslant g_{1} \Delta_{2}$. But this is an overcount, since we count $x^{\prime} \in N_{2}\left(f^{s}(u)\right)$ for each $u \in C$. With this observation we obtain

$$
|P| \leqslant g_{1} \Delta_{2}-|C|+1 \text {. }
$$

Moreover, by definition, $V\left(G-H_{2}^{S}\right) \subseteq P$.

Suppose $v=z$. Then $z u \in E_{1}$ and $\varphi(z) \varphi(u)=x^{\prime} f^{s}(u) \in E_{2}$. Then $z \in Q$, where

$$
Q:=\bigcup_{u^{\prime} \in N_{2}^{+}\left(x^{\prime}\right)} N_{1}\left(\left(f^{s}\right)^{-1}\left(u^{\prime}\right)\right) .
$$

Since $N_{2}^{+}\left(x^{\prime}\right) \leqslant g_{2}$ and $\mid N_{1}\left(\left(f^{s}\right)^{-1}\left(u^{\prime}\right) \mid \leqslant \Delta_{1}\right.$ for all $u^{\prime} \in V$, we have $|Q| \leqslant g_{2} \Delta_{1}$. Again, this is an overcount, since we count $x$ for each $u^{\prime} \in f^{s}(C)$. Thus

$$
|Q| \leqslant g_{2} \Delta_{1}-|C|+1 \text {. }
$$

Note that $x^{\prime} \in P$ and $x \in Q$, since $C \neq \emptyset$. Thus

$$
|P \cup f(Q)| \leqslant g_{1} \Delta_{2}+g_{2} \Delta_{1}-2|C|+2-1<n-|C| .
$$

It follows that there exists $z^{\prime} \in V-(P \cup f(Q) \cup f(C))$. By definition, $z^{\prime} \in V\left(H_{2}^{s}\right)$. So $z:=$ $f^{-1}\left(z^{\prime}\right)$ is good, and thus $f^{s+1}:=\varphi_{z}$ is a packing of $H_{1}^{s+1}$ with $H_{2}^{s+1}$. This completes the induction step and the proof.

\section{Conclusion}

Recall from Section 2 that there is a polynomial-time algorithm for determining $\operatorname{col}(G)$. Given the many applications of the 2-colouring number, it is natural to ask the following.

Problem 1. Is there a polynomial-time algorithm for calculating $\operatorname{col}_{2}(G)$ ?

In [14] it is shown that $\operatorname{gcol}(G)$, and thus also $\operatorname{col}_{2}(G)$, cannot be bounded in terms of $\chi_{a}(G)$. A positive answer to the next problem would provide another application of game colouring number to a non-game problem. 
Problem 2. Can $\operatorname{col}_{2}(G)$ and/or $\chi_{a}(G)$ be bounded in terms of $\operatorname{gcol}(G)$ ?

In light of Theorem 4.2 it would be interesting to find a good bound on $\operatorname{gcol}(G)$ in terms of $\theta(G)$. Here is a start. If $G$ is regular then $\operatorname{gcol}(G)=\frac{1}{2} \theta(G)+1$. So suppose $G$ is not regular. The high-degree vertices $v$ satisfying $d(v)>\frac{1}{2} \theta(G)$ form an independent set. Only these vertices can be adjacent to more than $\frac{1}{2} \theta(G)$ previously chosen vertices. So Alice will always choose high-degree vertices, if possible. Similarly, Bob will only choose low-degree vertices $v$ satisfying $d(v) \leqslant \frac{1}{2} \theta(G)$ which threaten unchosen high-degree vertices. We may assume that the low-degree vertices also form an independent set.

Proposition 6.1. For every positive integer $k \geqslant 3$ there exists a graph $G$ with

$$
k+1=\Delta(G)+1=\operatorname{gcol}(G)=\frac{\theta(G)+3}{2} .
$$

Proof. Fix $k$. Let $G$ be an $X, Y$-bigraph, where

$$
X:=\left\{x_{i, j}: i \in[k-1], j \in[k]\right\} \quad \text { and } \quad Y:=\left\{y_{j}: j \in[k]\right\} \cup\left\{z_{i, j}: i \in[k-1], j \in[k]\right\} .
$$

Define $E:=E(G)$ by

$$
E:=\left\{y_{j} x_{i, j}: i \in[k-1], j \in[k]\right\} \cup\left(z_{i, j} x_{i, h}: i \in[k-1], j, h \in[k], j \neq h\right\} .
$$

Then every vertex in $X$ has degree $k$ and every vertex in $Y$ has degree $k-1$. So $\operatorname{gcol}(G) \leqslant k+1$ and $\theta(G)=2 k-1$. Thus it suffices to show that Bob has a strategy for obtaining a score of at least $k+1$.

While possible, Bob will always choose vertices from $Y$, and as above, we may assume that Alice always tries to choose a vertex from $X$. Bob starts and begins by choosing $y_{1}, \ldots, y_{k}$. After Alice's first $k$ moves, there exists $i \in[k-1]$ such that at most one vertex of $X_{i}:=x_{i, 1}, \ldots, x_{i, k}$ has been chosen. Without loss of generality, no vertex of $X_{i}$, other than possibly $x_{i, k}$, has been chosen. Bob chooses $z_{i, k}$. From now on, whenever Alice chooses a vertex $x_{i, j}$ Bob chooses a vertex $z_{i, h}$ with $h=j$ if possible. When Alice chooses the last vertex of $X_{i}$, say $x_{i, n}$, each of its neighbours in the set $\left\{z_{i, j}: j \in[k]\right\}+y_{j}-z_{i, n}$ will already be chosen.

Proposition 6.2. Suppose that $G$ is an $X, Y$-bigraph such that

$$
d(y)=d \leqslant D=d(x) \text { for all } y \in Y, x \in X .
$$

Then $\operatorname{gcol}(G) \leqslant D+2-\left\lfloor\frac{D}{d}\right\rfloor$.

Proof. Let $k=\left\lfloor\frac{D}{d}\right\rfloor$. Obtain an $X^{\prime}, Y$-bigraph $G^{\prime}$ from $G$ by splitting each vertex $x \in X$ into $k$ vertices $x_{1}, \ldots, x_{k}$ of degree $d$ or $d+1$ so that $\left\{N\left(x_{i}\right): i \in[k]\right\}$ partitions $N(x)$. By Hall's Theorem, $G^{\prime}$ has a matching that saturates $X^{\prime}$. Recombining the vertices $x_{1}, \ldots, x_{k}$ into $x$ yields a set $\left\{S_{x}: x \in X\right\}$ of disjoint stars, where $S_{x}$ has centre $x$ and $k$ edges.

Whenever Bob chooses a vertex $y$ in a star $S_{x}$, Alice responds by choosing $x$ if it has not already been chosen. This ensures that when $x$ is finally chosen it will have at most $D-k+1$ previously chosen neighbours. 
Problem 3. Does every graph $G$ satisfy $\operatorname{gcol}(\mathrm{G}) \leqslant \frac{\theta(\mathrm{G})+3}{2}$ ?

\section{Dedication}

Professor Trotter has had a profound influence on our careers as well as the careers of many other mathematicians. It is a pleasure to honour him on the occasion of his 65 th birthday and wish him many happy returns of the day.

\section{References}

[1] Bodlaender, H. L. (1991) On the complexity of some coloring games. Internat. J. Found. Comput. Sci. 2 133-147.

[2] Bollobás, B. and Eldridge, S. E. (1978) Packing of graphs and applications to computational complexity. J. Combin. Theory Ser. B 25 105-124.

[3] Bollobás, B., Kostochka, A. V. and Nakprasit, K. (2005) On two conjectures on packing of graphs. Combin. Probab. Comput. $14723-736$.

[4] Burr, S. A. and Erdős, P. (1975) On the magnitude of generalized Ramsey numbers. In Infinite and Finite Sets (A. Hajnal, R. Rado and V. T. Sós, eds), Colloq. Math. Soc. Janos Bolyai, Vol. 1, North-Holland.

[5] Catlin, P. A. (1974) Subgraphs of graphs I. Discrete Math. 10 225-233.

[6] Chen, G. and Schelp, R. H. (1993) Graphs with linearly bounded Ramsey numbers. J. Combin. Theory Ser. B 57 138-149.

[7] Chvatál, V., Rödl, V., Szemerédi, E. and Trotter, W. T. (1983) The Ramsey number of a graph of bounded degree. J. Combin. Theory Ser. B 34 239-243.

[8] Dinski, T. and Zhu, X. (1999) A bound for the game chromatic number of graphs. Discrete Math. 196 109-115.

[9] Faigle, U., Kern, W., Kierstead, H. A. and Trotter, W. T. (1993) On the game chromatic number of some classes of graphs. Ars Combin. 35 143-150.

[10] Gardner, M. (1981) Mathematical games. Scientific American (April 1981), 23.

[11] Kierstead, H. A. (2000) A simple competitive graph coloring algorithm. J. Combin. Theory Ser. B 78 57-68.

[12] Kierstead, H. A., Mohar, B., Špacapan, S., Yang, D. and Zhu, X. The two-coloring number and degenerate colorings of planar graphs. Submitted.

[13] Kierstead, H. A. and Trotter, W. T. (1994) Planar graph coloring with an uncooperative partner. J. Graph Theory 18 569-584.

[14] Kierstead, H. A. and Trotter, W. T. (2001) Competitive colorings of oriented graphs. Electron. J. Combin. 8 \#12.

[15] Kierstead, H. A. and Tuza, Z. (2003) Marking games and the oriented game chromatic number of partial k-trees. Graphs Combin. 19 121-129.

[16] Kierstead, H. A. and Yang, D. (2003) Orderings on graphs and game coloring number. Order $20255-264$.

[17] Kostochka, A. V. and Yu, G. (2007) An Ore-type analogue of the Sauer-Spencer theorem. Graphs Combin. 23 419-424.

[18] Nešetřil, J. and Ossona de Mendez, P. (2008) Grad and classes with bounded expansion I: Decompositions. Europ. J. Combin. 29 760-776.

[19] Nešetřil, J. and Sopena, E. (2001) On the oriented game chromatic number. Electron. J. Combin. 8 \#14.

[20] Sauer, N. and Spencer, J. (1978) Edge disjoint placement of graphs. J. Combin. Theory Ser. B 25 295-302. 
[21] Zhu, X. (1999) Game coloring number of planar graphs. J. Combin. Theory Ser. B 75 $245-258$.

[22] Zhu, X. (2000) The game coloring number of pseudo partial $k$-trees. Discrete Math. 215 $245-262$.

[23] Zhu, X. (2008) Refined activation strategy for the marking game. J. Combin. Theory Ser. B 98 $1-18$.

[24] Zhu, X. Colouring graphs with bounded generalized colouring number. Discrete Math., to appear. 